\title{
IDC Robocon: A Transnational Teaming Competition for Project-Based Design Education in Undergraduate Robotics
}

\author{
Ning Tan ${ }^{1, *}$, Rajesh Elara Mohan ${ }^{2}$, Shaohui Foong ${ }^{2}$, Masaki Yamakita ${ }^{3}$, Masami Iwase 4 , \\ Shoshiro Hatakeyama ${ }^{4}$, Norihiro Kamamichi ${ }^{4}$, Libo Song ${ }^{5}$, You Wang ${ }^{6}$ and Qiuguo Zhu ${ }^{6}$ \\ 1 Advanced Robotics Centre, National University of Singapore, Singapore 117580, Singapore \\ 2 Pillar of Engineering Product Development, Singapore University of Technology and Design, \\ Singapore 487372, Singapore; rajeshelara@sutd.edu.sg (R.E.M.); foongshaohui@sutd.edu.sg (S.F.) \\ 3 Department of Mechanical and Control Engineering, Tokyo Institute of Technology, Tokyo 152-8552, \\ Japan; yamakita@ac.ctrl.titech.ac.jp \\ 4 Department of Robotics and Mechatronics, Tokyo Denki University, Tokyo 120-8551, Japan; \\ iwase@fr.dendai.ac.jp (M.I.); sho@fr.dendai.ac.jp (S.H.); nkama@fr.dendai.ac.jp (N.K.) \\ 5 SJTU Engineering Training Center, Shanghai Jiao Tong University, Shanghai 200240, China; \\ lbsong@sjtu.edu.cn \\ 6 College of Control Science and Engineering, Zhejiang University, Hangzhou 310027, China; \\ king_wy@zju.edu.cn (Y.W.); qgzhu@iipc.zju.edu.cn (Q.Z.) \\ * Correspondence: tanningrobotics@gmail.com; Tel.: +65-6499-4580
}

Academic Editor: Huosheng $\mathrm{Hu}$

Received: 2 April 2016; Accepted: 22 June 2016; Published: 24 June 2016

\begin{abstract}
This paper presents a robot design competition called 'IDC Robocon' as an effective tool for engineering education. The International Design Contest (IDC) Robocon competition has several benefits in creating a meaningful design experience for undergraduate engineering students and includes an international flavour as participants of the competition hail from all around the world. The problem posed to the contestants is to design, build and test mobile robots that are capable of accomplishing a task. A primary goal of the competition is to provide undergraduates with a meaningful design experience with an emphasis on mechanical design, electronic circuits and programming. It is hoped that by placing the emphasis on the design, the course will encourage more undergraduates to go into the field of engineering design. This paper presents the latest 2015 IDC Robocon (the 26th edition) in detail and discusses course of events and results in terms of the educational experience. In this competition, a simulated space problem of cleaning the debris from orbit is proposed for the latest IDC Robocon competition. Teams, comprising of students from multiple countries work together to develop robotic systems to compete with each other in collecting the foam balls and delivering them to the rotating the holder.
\end{abstract}

Keywords: IDC Robocon; robot competition; project-based; robotics education

\section{Introduction}

\subsection{Robotics Competitions}

In the last decades, there have been a lot of international competitions as a powerful means to push the state of the art in robotics research and development. RoboCup is the largest robotics competition attended by thousands of researchers every year comprising of individual focused leagues namely: (i) RoboCup@Home for developing domestic service robots using a so-called "system benchmarking" that evaluates robot performances in a realistic, complex and dynamic 
environment whose design focuses on exhibiting a high degree of uncertainty [1]; (ii) RoboCup Soccer for developing and benchmarking cooperative multi-robot and multi-agent systems in dynamic adversarial environments with the ultimate goal of building a humanoid soccer team that defeats the human World Cup Champion team by mid-century [2]; and (iii) RoboCup Rescue for developing and benchmarking physical/virtual robotic agents, information infrastructures and strategies for search and rescue missions with the purpose of helping mitigate the suffering of people from disasters [3]; (iv) RoboCupJunior aims to promote STEM content and skill learning among participating youth aged 19 or below through educational robotics competition inaugurated in 2000 [4]. Participants compete in one of three main leagues: Soccer, Rescue or Dance. RoCKIn is a three-year EU project that aims to use robot competitions in order to innovate smarter, more dependable robots, and to increase public awareness of robotics. RoCKIn@Work focuses on how robots could help European industry in the future [5], while RoCKIn@Home looks at developing domestic service robots [6]. The AAAI Robot Challenge [7] comprises of a benchmarking competition for human robot interaction that involves paper presentation by participating robots and a second benchmarking competition for search and rescue robots. The U.S. Defense Advanced Research Projects Agency (DARPA) sponsored some autonomous vehicle competitions [8] wherein the DARPA Grand Challenge [9] aims at developing and benchmarking autonomous robotic cars with a larger objective of minimizing traffic fatalities. Eurobot is a challenge that involves development and benchmarking of autonomous robots that collect artifacts on a defined playing arena with an evolving set of rules [10]. The DARwIn-OP Humanoid Application Challenge was a competition that focused on development and benchmarking of vision-aided humanoid robots. Other robot challenges [11] that have been carried out during ICRA conferences include: the Mobile Manipulation Challenge, a competition to show off the state of the art in integrated perception and manipulation; the Humanitarian Robotics and Automation Technology Challenge, a competition to benchmark applied robotics and automation technologies in solving problems related to humanitarian causes; and the Mobile Microrobotics Challenge, a competition focusses on testing the autonomy and mobility of small-scaled robots. World Robot Olympiad organizes robotics competitions in four different competition categories namely regular, open, soccer and advanced [12]. Another popular event is the FIRST Robotics Competition wherein teams of students and their mentors work towards solving a common problem over a period of six weeks using a standard set of components and a common set of rules [13].

Instead of focusing on education, these competitions are platforms for developing and benchmarking technologies and skills [14]. Few of them place emphasis upon robotics education aspects which have been a popular discipline because robotics can merge mechanical, electrical, electronic, and computer engineering [15,16]. Meanwhile, competitions are a commonly-used way in engineering education $[17,18]$. Especially for robotics education, such kinds of competitions are usually problem-based [19-21] and design-oriented [22-24] in a university setting. There is another type of extramural robot contest called ABU (Asia Broadcast Union) Asia-Pacific robot contest. However, the games in the contest are competed among teams from each country.

\subsection{IDC Robocon}

The history of IDC Robocon can be traced from a lecture called "creative design" at a department of control engineering (now control and systems engineering) in Tokyo Institute of Technology, which had been conducted by Prof. Mori from 1982. In that lecture, in order to activate creative nature of each student, "Zazen" which was a special style for meditation was introduced in the beginning of each lecture. In order to enhance the policy of the lecture, two lectures, called "Creative Design I" and "Creative Design II" were started from 1989 and a robot competition was held at the end of the Creative Design I. On the other hand, the Massachusetts Institute of Technology (MIT) had a similar lecture with a competition using robots for creative design in a department of mechanical engineering. Two universities acknowledged that they had very similar lectures and discussed a joint activity to enhance the function of the lectures by having an international design contest, which was 
IDC, from 1990. The latest IDC Robocon 2015 was the 26th instalment which was organized by Singapore University of Technology and Design (SUTD) in Singapore. For more background about IDC Robocon, readers are referred to $[25,26]$.

As for many other design education events [27,28], IDC RoboCon is also a team-based design competition where university students from all parts of the world gather to share ideas and knowledge on robotics and develop winning robots. The objective of the competition is to foster and develop creativity, technique and international experience of young future engineers. Every year, the contest is hosted in rotation by the member of the IDC Robot Contest Organizing Committee consisting of universities around the world. Coming from different cultures and backgrounds, and working in mixed groups, the students have to overcome language and cultural differences to design and build a robot, within two weeks, to accomplish a task. The following points make IDC Robocon particularly interesting:

1. Universities are increasingly embracing project-based learning with in-built competitive elements. Robocon seeks to introduce this competitive element to students who may not have encountered it before.

2. Competitive robotic challenges normally pit teams from same university against each other. Robocon seeks to promote diversity and understanding across cultures/race/religion.

3. The theme is not announced to participants until the actual challenge, so no team gets any advantage.

Section 2 below discusses the pedagogical issues and spirits of the IDC Robocon competition. Section 3 presents the organization aspects of the newest implementation of the competition. Section 4 presents the results of the competition and discusses the related experience. Finally, the paper is concluded in Section 6.

\section{Unique Pedagogical Approaches}

The IDC Robocon competition serves as an education medium rather than a benchmarking platform like most robot competitions. A persistent problem facing today's engineering educators is how to promote and retain students' interest in science and engineering beyond the conventional lecture, tutorial and laboratory setting. It is becoming an increasing necessity with more and more engineering graduates switching to non-engineering careers upon graduation. One of the best approaches to this challenge is to promote student participation in a technological competition through publicity and the spirit of competitiveness as well as the attraction of being exposed to students all around the world.

\subsection{Fast Learning}

IDC Robocon seeks to cultivate the fast learning capability in two weeks. Prior to coming to the organization institution, the students and their leading advisors have no idea of the challenge theme and whom he/she will be assigned to. The students coming from different cultures and backgrounds work together in mixed groups that are designed to promote diversity. Within two weeks, they have to overcome language and cultural differences to design and build a robot to accomplish a task in keeping with the philosophy of learning by doing and principles of problem-based or project-based learning. Since the theme is not announced to participants until the actual challenge, no team gets any advantage.

\subsection{Project-Based and Competitive Engineering Education}

Educatiors in universities are increasingly embracing project-based learning with in-built competitive elements [29]. Robocon seeks to introduce this competitive element to students who may not have encountered it before. Through setting a appropriate problem, the competition merges the expertise of mechanism design, rapid prototyping, electronics, path tracking, control, etc. Besides, 
the competition also accepts participation of a few high school students. Then they have a chance to learn and work with the senior students and advisors, thereby discovering the interests and potential themselves.

\subsection{Transnational Teaming}

One of the most typical features and merits of the IDC Robocon competition is transnational teaming where every team consists of students from different countries. Competitive robotic challenges normally pit teams from the same university against each other. Robocon seeks to promote diversity and understanding across cultures, races and religions. The students from the same countries are assigned to different teams so as to maximize the diversity of each team. This reduces the pressure on individual students if they were competing for their respective countries.

Such teaming can enhance cultural exchange and diversity which gives undergraduate students a new experience, especially for those from the countries with fewer international students, such as China, Japan, Egypt, etc. During the competition, contestants from multinational teams work together and build robots beyond cultural differences and language barriers. Since the curriculum systems of engineering education are various in different countries, the teammates can complement based on their skill sets and knowledge.

On the other hand, if every team were formed by members from the same country, the antagonism of the competition would be much higher. This teaming plays down the antagonism but enhances cultural and knowledge exchange. Then the students can be more focused on creativity and communication rather than victory. So this competition is more like a project-based summer school where multinational students get together and are divided into different teams randomly, and then work on a given project. In short, this teaming balances the antagonism, creativity and collaboration. These kind of experiences should be important for students who will be international researchers or engineers in a worldwide business situation.

\subsection{Multidisciplinary Teaming}

The IDC Robocon competition normally requires each team to build two or more robots to finish the project. Students should have knowledge of mechanics, electronics, control, computers, etc. Some universities, such as Zhejiang University, select most students from engineering majors to join the IDC Robocon. Students with different background are divided into different teams randomly. Probably in one team, all the students are good at mechanics, but nobody can program microcontrollers. The students have to learn fast and have good teamwork. Communications with other engineering majors can extend the scope of students' knowledge, which is the most charming point when developing the robots. Efforts are being made to run a pre-competition survey to collect technical background of individuals participating in the competition so as to use this information for formation of more balanced teams in the 2016 run of the event.

\subsection{Transnational Mentorship}

Since the students from a same country are distributed evenly to diverse groups, their leading advisors (formed by professors, lecturers, researchers) can give technical advise and comments freely and unreservedly without considering the outcome of the game, even if some of them are from other countries. This makes mentorship much more comfortable and enjoyable. In addition, the advisor from one country can learn from other advisors through discussing and exchanging experience. Then he or she would be able to grab valuable things from other universities' curriculum systems, course contents, and assessment methods, and then combine this with their school features to bring it into the teaching and practice systems. 


\section{IDC Robocon 2015: Space Cleaner}

In this section, the IDC Robocon 2015 will be presented as a case study following the pedagogical approaches introduced above. The theme chosen for the Robocon 2015 event was Space Cleaner. The space theme was chosen due to renewed interest and the increased number of actual space missions witnessed globally in 2014-2015. The details of the theme, briefing on rules and the team formation were done during the one hour introduction session on the first day of the competition. No prior information on the theme or contest rules were made available to the participants and faculty representatives. The introduction session also included site visits to the contest venue for the teams to better understand the context. Any queries on the theme and/or rules were handled during the daily team leader meetings.

\subsection{Context and Objective}

Beginning in 1957 with the launch of the satellite Sputnik by the Soviet Union, humanity began to shoot for the stars. However, with this journey there also came space debris-defunct objects orbiting around the Earth. These objects are a potential hazard for the functioning satellites and people stationed in space. It is estimated that there are 21,000 pieces of space debris larger than 10 centimetres in orbit, and half a million pieces that are $1-10 \mathrm{~cm}$. These numbers are predicted to increase. In addition, there are also millions of pieces of debris smaller than 1 centimetre. Hence there is growing motivation to address these space debris so that future space launches will not be compromised.

In the lower parts of orbit around Earth, objects travel at $7 \mathrm{~km} / \mathrm{s}$. At this speed, a tiny speck of paint has the same effect as a $250 \mathrm{~kg}$ object traveling at $97 \mathrm{~km} / \mathrm{h}$. Not only can these small particles damage important modules such as pressurized items and tethers, but they can also create new debris upon impact. According to NASA, an average of one cataloged piece of debris has fallen back to Earth each day for the past 50 years. In recent years, various space organizations have reduced the amount of trash added to Earth's orbit by implementing better designs. However, cleaning the debris from orbit is still a problem that needs to be addressed. There have been plans of taking trips to larger objects to remove them from orbit, but this comes at a high financial cost.

The teams will design and built robots to remove the space debris from orbit and return the debris back to earth. The participated universities (country) includes SUTD (Singapore), MIT (USA), Shanghai Jiao Tong University (China), Tsinghua (China), Zhejiang University (China), Seoul National University (Korea), Menofia University (Egypt), Tokyo Institute of Technology and Tokyo Denki University (Japan), AbdelMalek Essaadi University (Morocco), and five universities from Thailand. In addition, seven students from Singapore's junior colleges, high schools and polytechnics participated the competition as well.

\subsection{General Guidelines}

The general guidelines are presented here.

1. The rules are intended to create opportunities to innovate and test new ideas which are fundamental to gaining confidence as an Engineer.

2. The IDC Robocon referees and staff make final scoring judgement calls and interpretations of the rules.

3. Every day, there is be a compulsory team leaders' meeting, and at least one representative from each team has to be present. Any discussion and decision on the rules are made during this meeting.

4. Robots must be constructed with materials provided in a kit and staff in a workshop, except extra purchases of about 20 US\$.

5. In a workshop at least one instructor should be present to monitor safety and consult with the students about design and construction of machines. 


\subsection{Game Field}

The game field is designed to be dedicated to the competition with following specifications.

1. The game field is consists of three zones: The outer orbit where the debris are present, the space across which the debris have to be transported and the earth which is the final destination for the debris. The detailed dimensions and views are given in Figure 1a-c.

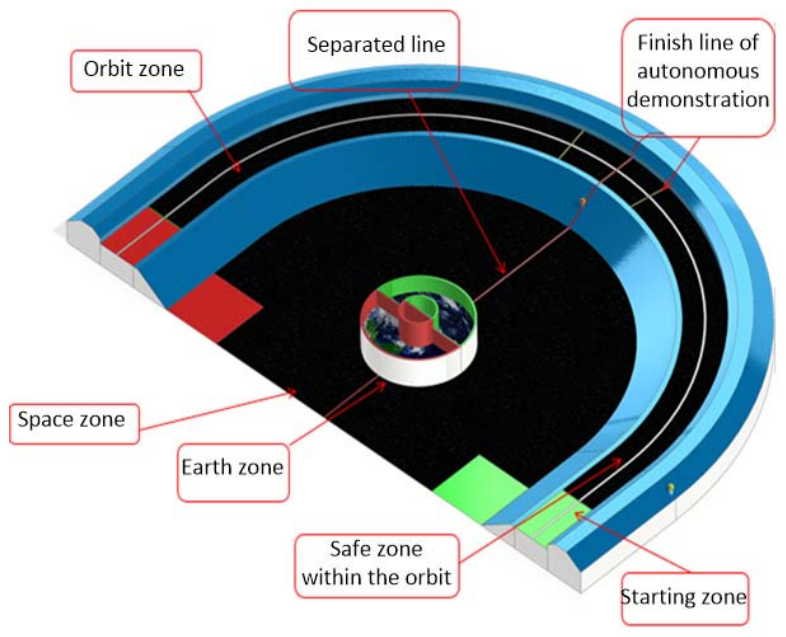

(a) Isometric view

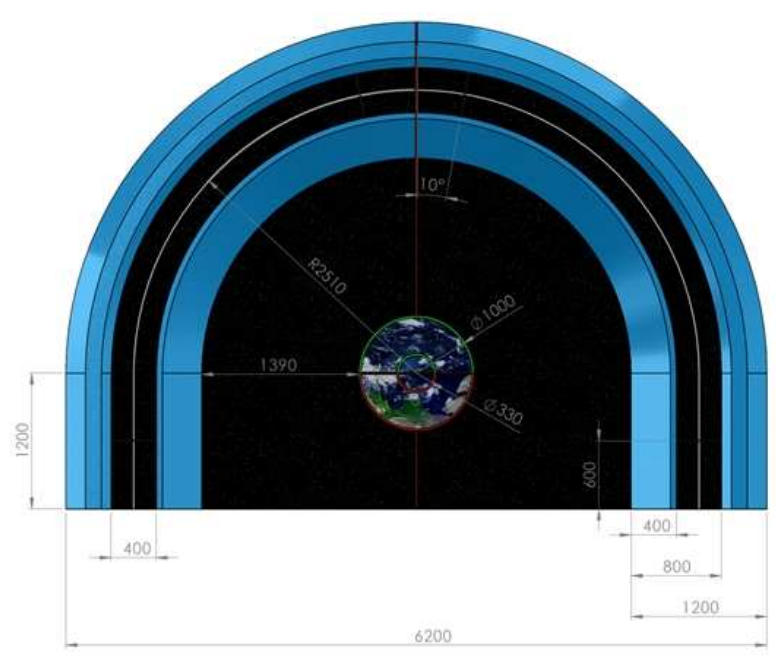

(b) Top view

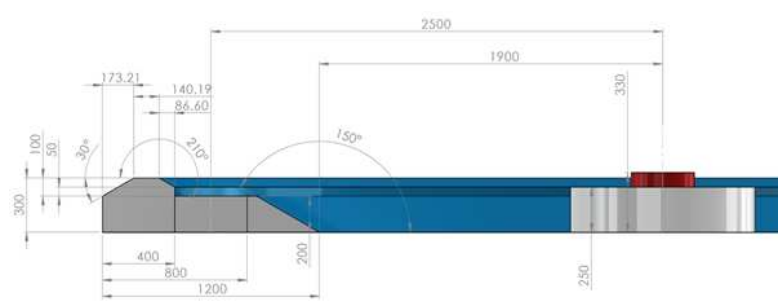

(c) Side view

Figure 1. CAD design of the contest campus. (a) Isometric view; (b) Top view; (c) Side view.

2. Earth on the game field rotates to simulate the orbiting space debris. 
3. Earth consists of dedicated regions for each team to deposit the debris collected by the robots for scoring points which is further divided into two areas-an outer shell area and a more elevated but smaller inner shell area.

4. Deployment of robots is restricted to a dedicated safe zone within the orbit and the entire space zone.

5. The entire arena is split into two parts, with one for each team and the crossover of robots are strictly not allowed. Any robot that enters the opponent's arena would be taken out of the field for the rest of the game.

6. The starting point of the robot located in space zone is a $600 \mathrm{~mm} \times 600 \mathrm{~mm}$ square beside the orbit zone and the outer side boundary of the space zone. The starting point of the robot located in the orbit zone is a $400 \mathrm{~mm} \times 600 \mathrm{~mm}$ rectangle beside the terminal edge of the orbit zone.

\subsection{Objects}

This subsection depicts the debris objects.

1. There are three types of debris objects within the game field, colored in white, yellow and orange, with varying points allocated to each. The debris are foam balls with a diameter of $50 \mathrm{~mm}$.

2. There is a total of 81 pieces of debris -40 of them are scattered in each of the two regions dedicated for the teams and one orange piece of debris located at the boundary line between the regions. Out of the 40 pieces of debris located within each region, 12 of them are yellow, and 28 of them are white. The locations of the pieces of debris are illustrated in Figure 2. The yellow-colored area is where yellow debris is located, and the white area is where the white debris is located. The orange point is where the orange debris is located.

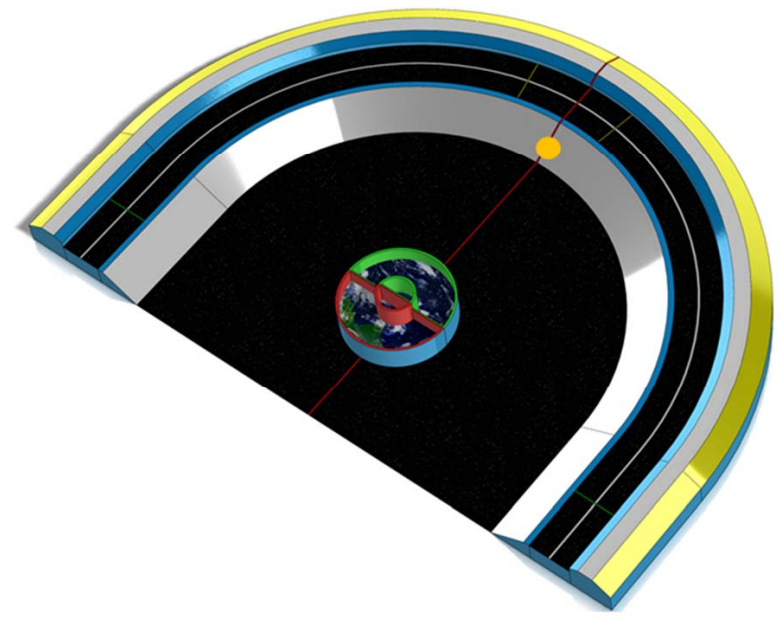

Figure 2. Locations of three kinds of debris.

3. Debris that fall off from the game field are out of play.

4. The debris are located on the top of a diameter $30 \mathrm{~mm}$ tube that is inserted the foam.

\subsection{Robot}

The robots to be developed must fit some specifications which are clarified as follows.

1. Each team is allowed a maximum of two robots.

2. The entire robot must be made from the kit materials and materials purchased using the funds (SGD 50) allocated for each team. All components purchased out of the kit must be authorized by one of the official referees. Screws, bolts, nuts, washers, stick glues, vinyl tape, welds and many other materials are prepared for the teams at the workplace. The list of components in the kit is given in Table 1. 
3. The maximum allowable robot size is $600 \mathrm{~mm} \times 600 \mathrm{~mm} \times 600 \mathrm{~mm}$. Robots can be designed with active deployable mechanisms with no size restrictions.

Table 1. List of Components in Kit.

\begin{tabular}{clc}
\hline S/N & Description & Oty (per Team) \\
\hline Electronics & 1 \\
\hline 1 & DFR0305 Romeo BLE & 1 \\
2 & DFR COMB0003 DFRduino Mega Kit for 4 motor robot & 1 \\
3 & Turnigy 9X 9Ch Transmitterw / Module \& 8Ch Receiver & 2 \\
4 & LiPo Battery & 1 \\
5 & Micro USB cable & 1 \\
6 & Printer port USB cable & 2 \\
\hline Actuators & 3 \\
\hline 1 & SRC SM-S4303R Continuous RotationServo & 2 \\
2 & HXT 900 Micro Servo & 2 \\
3 & Turnigy metal gear micro servo TSS-10MG & 1 \\
4 & Turnigy TGY-R5180MG 180 Degree MetalMini Servo & 1 \\
5 & VSD-11Y 360 Degree Large Servo & 2 \\
6 & Geared DC Motor, 133 rpm, 75:1,6 V & 1 \\
7 & HXT 12K 10 kg Servo & 1 \\
8 & 28BYJ-48-5V 4-Phase 5-Wire Stepper Motor Kit & \\
9 & SW5513-4MA Sailwinch Servo 10.63 kg/55 g/0.9 & 1 \\
\hline Wheels & \\
\hline 1 & POL226-5/8 inch plastic black wheel for continuous servo & \\
\hline Accessories & \\
\hline 1 & 4WD Vehicle Kit (4 DC motors) & 2 \\
\hline
\end{tabular}

4. The total weight of each robot must not exceed $5 \mathrm{~kg}$.

5. The machines in the fabrication lab, for example bench drilling machines, band saws, benders, 3D printers, laser cutters and hand tools, can be used. The technical facilitators in the fabrication lab and IDC Robocon staff can be asked on how to access them.

6. Each robot must be defined either to operate in orbit or space zones and they are expected to stay in their respective zone for the duration of the match. The robot in orbit must stay in orbit zone and the robot that is deployed in the space must stay within that area at all times.

7. Teams can decide to put one robot in orbit and space zone or both robots in one of the two zones.

8. For robots that fall off the defined area, team members are allowed to place them back onto the field to restart from the starting area. However, a penalty is levied.

\subsection{Scoring}

The scoring system is stipulated as follows.

1. To score, the team must transfer the debris from the orbit zone back to their respective regions on earth. The debris are color-coded and the point value of each colored debris is listed below in Table 2.

2. When the team deposits the debris into the outer shell area of the Earth, they get the points listed in Table 2. For each piece of debris transferred into the smaller elevated inner shell of the Earth, it is worth three times the points allocated for that colored debris, except for the orange debris which doubles the total points when deposited anywhere into the team's earth region. 
Table 2. Points allocated for colored debris.

\begin{tabular}{cc}
\hline Color & Points \\
\hline Orange & Doubling total score \\
Yellow & 30 \\
White & 10 \\
\hline
\end{tabular}

3. Whenever a robot goes out of the respective play area defined and/or crashes on either sides of the outer orbit, or any part of the earth zone, a penalty of 120 points would be levied, and the robot has to restart from the starting area.

4. Teams that demonstrate an autonomous line following from starting area to the demarcated boundary line are awarded an additional 120 points. This demonstration has to be done within the allocated game play time for the team.

\subsection{Schedules and Timing}

The schedules and timing include the following main points:

1. The contest consists of qualifying rounds and a final competition.

2. The qualifying rounds take place on 31st of July morning $9 \mathrm{am}-12 \mathrm{pm}$.

3. The finals is on the 31st of July evening $4 \mathrm{pm}-6 \mathrm{pm}$.

4. The qualifying round is $150 \mathrm{~s}$, and final tournament is $180 \mathrm{~s}$.

5. If a game finishes in a tie, the following tie-breaking rule will be applied; a game of $30 \mathrm{~s}$ to collect a single orange debris placed on the boundary line. The team that collects and deposits the orange debris will win the tie. This tie-breaking rule will apply until a winner emerges.

\subsection{Control}

The behaviors of contestants are controlled in a certain scope in order to help grease the wheels for the competition. Some limitations are highlighted:

1. Damaging, overturning, pushing and lifting an opponent's robot are not allowed.

2. Damaging the game field and/or control equipment is strictly prohibited.

3. During the workshop time and competition time, participants are supposed to respect and protect the game field. Stepping, moving, jumping over the game field is strictly prohibited.

4. Contestants and/or spectators may not directly affect the motion of the robot.

5. After the time limit, the referee judges the winner of the match according to the points scored by the competing teams.

6. No dangerous machines are allowed. Technical facilitators and IDC Robocon staff's decisions on safety must be respected and obeyed at all times.

7. Team members controlling the robot must wear safety glasses near the game field. Certain prescription glasses are acceptable.

\section{Results and Discussions}

After 20 round qualifying matches, 8 teams successfully moved into quarter finals. In the semi-finals stage, LIME OF HOPE, HONEY KUMQUAT, THE JANITORS and MEC-JT competed for the qualification of the final. A scenario of the event is given in Figure 3. Finally, HONEY KUMQUAT defeated MEC-JT and won the first prize.

Figure 4 demonstrates the robots developed and shown by the 10 teams in the competition. The principles and approaches adopted for ball fetching and collecting by the robots are summarized in Table 3. Most of the teams adopted the strategy that first the line-tracking robot fetches the balls on the orbit, and then the delivery robot collects the balls and delivers them to the central container. Unfortunately, Team 4 could not present their tracking robot and the delivery robot was not working, 
even though it finally started working for a long while after the game had started. The full results of the Robocon 2015 are presented in Table 4.

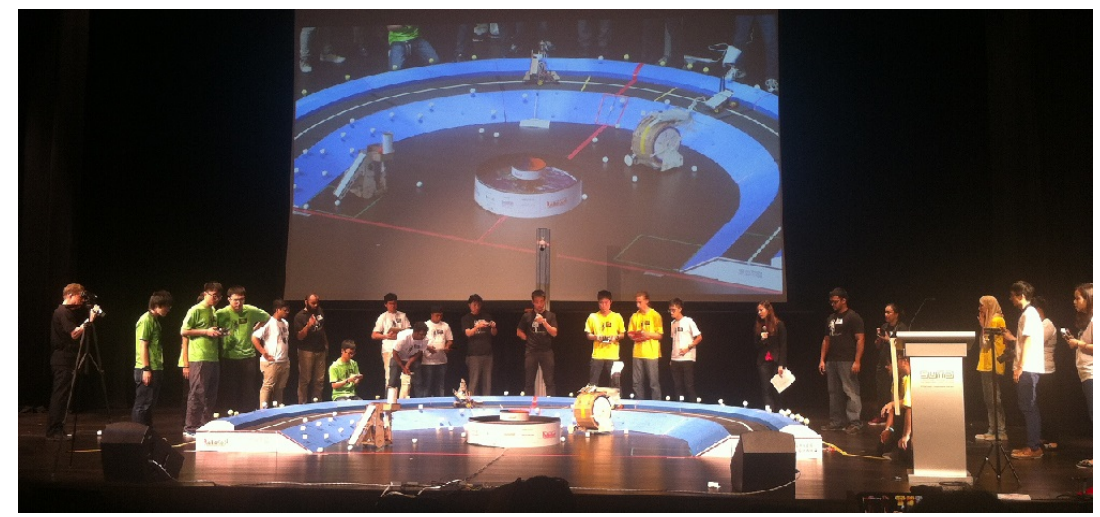

Figure 3. A scenario of the International Design Contest (IDC) Robocon 2015.

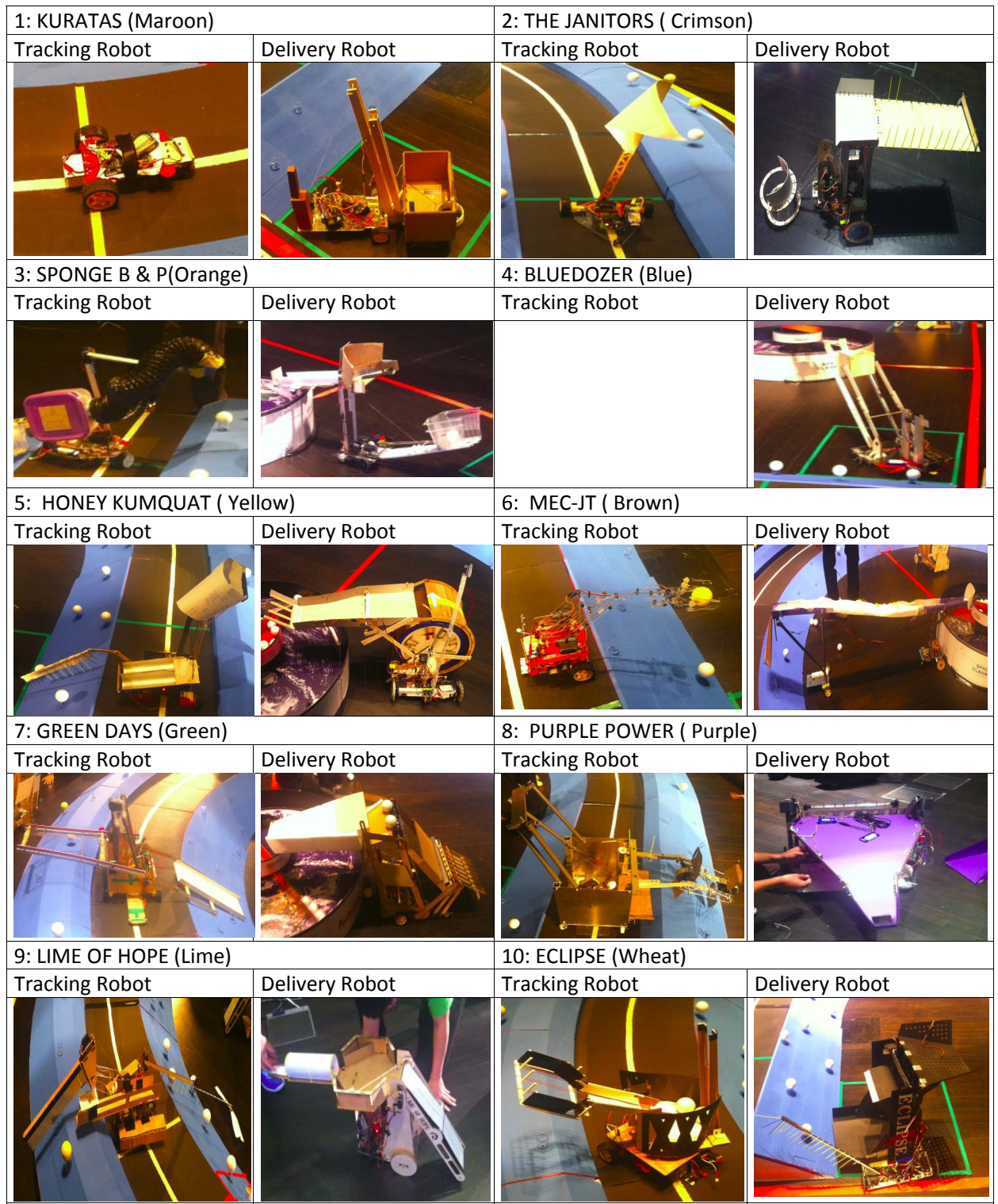

Figure 4. Robots designed and developed by the 10 teams. 
Table 3. Principles and approaches adopted for ball fetching and collecting by the robots.

\begin{tabular}{cccc}
\hline No. & Teams & Tracking Robots & Delivery Robots \\
\hline 1 & KURATA & NA & rubber band \\
2 & THE JANITOR & sweeping & rubber band \\
3 & SPONGE B \& P & pneumatic sucking & passive \\
4 & BLUEDOZER & NA & passive \\
5 & HONEY KUMQUAT & sweeping, rubber band & rubber band, roller \\
6 & MEC-JT & gripping & passive \\
7 & GREEN DAYS & sticking & sweeping, sticking \\
8 & PURPLE POWER & sweeping & sticking \\
9 & LIME OF HOPE & sweeping, ticking & sticking \\
10 & ECLIPSE & rubber band & sweeping \\
\hline
\end{tabular}

Table 4. Full Results of IDC Robocon 2015 Space Cleaner.

\begin{tabular}{|c|c|c|c|c|c|}
\hline Time & Match & $\begin{array}{c}\text { [LEFT] } \\
\text { ORANGE }\end{array}$ & Score & Score & $\begin{array}{l}\text { [RIGHT] } \\
\text { BLUE }\end{array}$ \\
\hline \multicolumn{6}{|c|}{ Qualifying Round Robin } \\
\hline $10: 30$ & 1 & KURATAS & 20 & -1 & BLUEDOZER \\
\hline $10: 40$ & 2 & GREEN DAYS & 0 & 180 & MEC-JT \\
\hline $10: 50$ & 3 & SPONGE B \& P & 0 & 400 & LIME OF HOPE \\
\hline $11: 00$ & 4 & PURPLE POWER & 0 & 0 & ECLIPSE \\
\hline $11: 10$ & 5 & BLUEDOZER & -1 & 30 & THE JANITORS \\
\hline $11: 20$ & 6 & MEC-JT & 150 & 0 & HONEY KUMQUAT \\
\hline $11: 30$ & 7 & KURATAS & 60 & 120 & SPONGE B \& P \\
\hline $11: 40$ & 8 & GREEN DAYS & 20 & 0 & PURPLE POWER \\
\hline $11: 50$ & 9 & LIME OF HOPE & 120 & 80 & THE JANITORS \\
\hline $12: 00$ & 10 & ECLIPSE & 0 & 420 & HONEY KUMQUAT \\
\hline $13: 00$ & 11 & SPONGE B \& P & 0 & -1 & BLUEDOZER \\
\hline $13: 10$ & 12 & PURPLE POWER & 0 & 270 & MEC-JT \\
\hline $13: 20$ & 13 & THE JANITORS & 460 & 0 & KURATAS \\
\hline $13: 30$ & 14 & HONEY KUMQUAT & 210 & 0 & GREEN DAYS \\
\hline $13: 40$ & 15 & BLUEDOZER & 0 & 280 & LIME OF HOPE \\
\hline $13: 50$ & 16 & MEC-JT & 240 & 120 & ECLIPSE \\
\hline $14: 00$ & 17 & THE JANITORS & 0 & 0 & SPONGE B \& P \\
\hline $14: 10$ & 18 & HONEY KUMQUAT & 180 & 60 & PURPLE POWER \\
\hline $14: 20$ & 19 & LIME OF HOPE & 210 & 240 & KURATAS \\
\hline $14: 30$ & 20 & ECLIPSE & 120 & 150 & GREEN DAYS \\
\hline \multicolumn{6}{|c|}{$\begin{array}{l}\text { Championship Bracket } \\
\text { Quarter Finals }\end{array}$} \\
\hline $16: 10$ & QF1 & SPONGE B \& P & 120 & 180 & HONEY KUMQUAT \\
\hline $16: 20$ & QF2 & THE JANITORS & 0 & 180 & GREEN DAYS \\
\hline $16: 30$ & QF3 & LIME OF HOPE & 0 & 400 & PURPLE POWER \\
\hline $16: 40$ & QF4 & KURATAS & 0 & 450 & MEC-JT \\
\hline \multicolumn{6}{|c|}{ Semi Finals } \\
\hline $16: 50$ & SF1 & LIME OF HOPE & 160 & 490 & HONEY KUMQUAT \\
\hline $17: 00$ & SF2 & THE JANITORS & 200 & 510 & MEC-JT \\
\hline \multicolumn{6}{|c|}{ Third Place \& Finals } \\
\hline $17: 10$ & $\mathrm{TP}$ & THE JANITORS & 280 & 360 & LIME OF HOPE \\
\hline $17: 20$ & GF & HONEY KUMQUAT & 720 & 360 & MEC-JT \\
\hline \multicolumn{6}{|c|}{ Final Results } \\
\hline \multirow{4}{*}{\multicolumn{2}{|c|}{$\begin{array}{l}\text { DESIGN PRIZE } \\
\text { THIRD PRIZE } \\
\text { SECOND PRIZE } \\
\text { FIRST PRIZE }\end{array}$}} & THE JANITORS & & & \\
\hline & & LIME OF HOPE & & & \\
\hline & & MEC-JT & & & \\
\hline & & HONEY KUMQUAT & & & \\
\hline
\end{tabular}


The line-tracking robots, on one hand, are responsible to reach the finish line through automatically tracking the line for autonomous demonstration. On the other hand, these robots are responsible for fetching balls on the orbit and passing to the delivery robots. One of the most frequently adopted strategies is that the tracking robots of Teams 1, 2, 5 and 9 swept the balls down using a configured limb. Another way to fetch balls is the adhesive approach whereby the robot used some adhesive bands to stick the balls so as to remove them from the orbit. The fetching principle of Team 3 is unique among all the teams. In the tracking robot, a vacuum sucking system is configured to suck the balls through a pneumatic tube. The installation consists of a ball pit and an pneumatic tube transport. When the intake approached the ball closely enough, the ball would be absorbed into the container by the sucking force generated by the built-in pump. Team 6 adopts a dexterous solution such that a motorized gripper is able to get the high-point balls (the yellow ones) which are difficult to reach and to fetch by sweeping. This means they can collect high-point balls but it is also relatively time-consuming compared to other means, which allowed them to enter the final but caused them to loose the champion. In the tracking robot, Team 10 designed a ball fetcher through tightening four sets of rubber bands in parallel. Due to the elasticity, once the fetcher clapped on the balls, they can enter the holder and be held by the robot.

On the delivery robot side, one of the most dexterous robots is from Team 2. It adopts the same principle as Team 10's tracking robot to collect the balls using rubber bands (Team 1 also employ such an approach). Thanks to its dexterity during the game, this robot can efficiently collect the balls on the floor. Team 5 won the first prize because of their powerful ball-collecting machine. The delivery robot of this team is very efficient at collecting balls through rotating a cylindrical mechanism like a roller. Even though its mobility is not the best, this machine can collect multiple on-ground balls at the same time. Being the second-prize winner, the two robots of Team 6 are distinctly different in their mobility. As mentioned before, the tracking robot has high manoeuvrability, but the delivery robot only passively received balls from its partner. Team 9 acquired the third prize because, particularly, they adequately exploit the adhesive force using adhesive tapes by which the tracking robot could fetch the yellow balls and the delivery robot could collect the balls on the ground.

During the competition, every team assigned tasks to each member according to his/her know-how and skills. For example, in most of the cases, Japanese students are responsible of controlling the robots using the hand control lever because they are used to playing computer games. The team members cooperatively developed the robots in virtue of their expertise in mechanism design, rapid prototyping, electronics, path tracking, control, etc.

\section{Lessons Learned}

\subsection{Problems in Students}

During the contest, we found several problems met by the students. First, few students could use CAD design softwares (e.g., Solidworks) which could very well be due to the lack of preceding practices; Second, because the power of the provided motors is relatively small, some students had to make plenty of modifications on their designs since they did not consider the maximum torques. The core reason for this is also due to the lack of practical experience; Third, since 2013, some students could not use Arduino very well to design a good control system which affected the final performances of the robots.

\subsection{Problems in Rules}

In most cases, the rules were designed, debugged, and revised simultaneously while a portion of rules were totally canceled and modified. However, due to limitations of time and materials, some rules were not able to be modified, and that had an influence on the final results. Besides, the teaming regulation was based on sortition before 2012. However, the appointing of teams has been adapted since 2013, which could cause some loss in randomness and interest. 


\section{Conclusions}

A transnational teaming competition, IDC Robocon, was adopted for project-based education in undergraduate robotics. The most distinct feature of IDC Robocon is that all participants from different countries are shuffled and regrouped to make a team with four students from different countries, and a different contest rule is given every year by a team composed of students from the same university or a country in the usual robot contests. There are three main merits that make Robocon different from other robot/engineering competitions. First, Robocon seeks to introduce the competitive element to students who may not have encountered it before in their college curriculum. Second, Robocon seeks to promote diversity and understanding across cultures, races and religions. Third, the theme is not announced to participants until the actual challenge. Thus, no team gets any advantage. In this paper, the full process of the latest 26th Robocon was presented. Based on the results, it can be learned that the transnational teaming triggered students' creativity in terms of strategies and robot designs. The prepared stage and finals fostered students' hands-on skills and understanding of curriculum knowledge.

Acknowledgments: The authors would like to thank Ruihe Cai for his organization and campus building.

Author Contributions: N.T., R.E.M. and S.F. conceived and designed the experiments; N.T. R.E.M. and S.F. performed the experiments; N.T. and R.E.M. analyzed the data; M.Y., M.I., S.H., N.K., L.S., Y.W. and Q.Z. contributed reagents/materials/analysis tools; N.T. and R.E.M. wrote the paper.

Conflicts of Interest: The authors declare no conflict of interest.

\section{References}

1. Wisspeintner, T.; van der Zant, T.; Locchi, L.; Schiffer, S. RoboCup@Home: Scientific Competition and Benchmarking for Domestic Service Robots. Interact. Stud. 2009, 10, 392-426.

2. Kitano, H.; Asada, M. The RoboCup humanoid challenge as the millennium challenge for advanced robotics. Adv. Robot. 1998, 13, 723-736.

3. Tadokoro, S. RoboCupRescue international research project. Adv. Robot. 2002, 16, 569-572.

4. Eguchi, A. RoboCupJunior for promoting STEM education, 21st century skills, and technological advancement through robotics competition. Robot. Auton. Syst. 2016, 75, 692-699.

5. Dwiputra, R.; Bonn-Rhein-Sieg, H.; Berghofer, J.; Ahmad, A.; Awaad, I.; Amigoni, F.; Bischoff, R.; Bonarini, A.; Fontana, G.; Hegger, F.; et al. The RoCKIn@Work Challenge. In Proceedings of the 41st International Symposium on Robotics, Munich, Germany, 7-9 June 2010.

6. Schneider, S.; Hegger, F.; Ahmad, A.; Awaad, I.; Amigoni, F.; Berghofer, J.; Bischoff, R.; Bonarini, A.; Dwiputra, R.; Fontana, G.; et al. The RoCKIn@Home Challenge. In Proceedings of the 41st International Symposium on Robotics, Munich, Germany, 7-9 June 2010.

7. Simmons, R.; Goldberg, D.; Goode, A.; Montemerlo, M.; Roy, N.; Sellner, B.; Urmson, C.; Schultz, A.; Abramson, M.; Adams, W.; et al. GRACE: An Autonomous Robot for the AAAI Robot Challenge. AI Mag. 2003, 24, 51-72.

8. Baker, C.R.; Dolan, J.M. Street Smarts for Boss. IEEE Robot. Autom. Mag. 2009, 16, 78-87.

9. Iagnemma, K.; Buehler, M. Special issue on the DARPA grand challenge, Part 1. J. Field Robot. 2006, 23, 461-462.

10. Eurobot. International Autonomous Robot Contest; Eurobot Association: Ris-Orangis, France, 2016.

11. Smart, B. The ICRA Robot Challenge. IEEE Robot. Autom. Mag. 2010, 17, 8-10.

12. Giannakopoulos, N. Chapter Experiences from WRO 2009 Competition and Verifications about the Robotics Incorporation in the School. In Book of Abstracts of the Conference: Lessons Learnt from the Terecop Project and New Pathways into Educational Robotics across Europe; TERECoP: Athens, Greece, 2009.

13. Melchior, A.; Cohen, F.; Cutter, T.; Leavitt, T.; Manchester, N.H. More than Robots: An Evaluation of the First Robotics Competition Participant and Institutional Impacts; Technical Report; Heller School for Social Policy and Management, Brandeis University: Waltham, MS, USA, 2005. 
14. Amigoni, F.; Bonarini, A.; Fontana, G.; Matteucci, M.; Schiaffonati, V. Benchmarking through competitions. In European Robotics Forum-Workshop on Robot Competitions: Benchmarking, Technology Transfer, and Education; European Robotics Forum: Lyon, France, 2013.

15. Cappelleri, D.J.; Vitoroulis, N. The Robotic Decathlon: Project-Based Learning Labs and Curriculum Design for an Introductory Robotics Course. IEEE Trans. Educ. 2013, 56, 73-81.

16. Jung, S. Experiences in Developing an Experimental Robotics Course Program for Undergraduate Education. IEEE Trans. Educ. 2013, 56, 129-136.

17. Telenko, C.; Wood, K.; Otto, K.; Elara, M.R.; Foong, S.; Pey, K.L.; Tan, U.X.; Camburn, B.; Moreno, D.; Frey, D. Designettes: An Approach to Multidisciplinary Engineering Design Education. J. Mech. Des. 2016, 138, doi:10.1115/1.4031638.

18. Battisti, F.; Boato, G.; Carli, M.; Neri, A. Teaching Multimedia Data Protection Through an International Online Competition. IEEE Trans. Educ. 2011, 54, 381-386.

19. Pack, D.J.; Avanzato, R.; Ahlgren, D.J.; Verner, I.M. Fire-fighting mobile robotics and interdisciplinary design-comparative perspectives. IEEE Trans. Educ. 2004, 47, 369-376.

20. Firebaugh, S.L.; Piepmeier, J.A. The RoboCup Nanogram League: An Opportunity for Problem-Based Undergraduate Education in Microsystems. IEEE Trans. Educ. 2008, 51, 394-399.

21. Chen, N. A Vision-Guided Autonomous Vehicle: An Alternative Micromouse Competition. IEEE Trans. Educ. 1997, 40, 253-258.

22. Yilmaz, M.; Ozcelik, S.; Yilmazer, N.; Nekovei, R. Design-Oriented Enhanced Robotics Curriculum. IEEE Trans. Educ. 2013, 56, 137-144.

23. Mohan, R.E.; Rojas, N.; Sosa, R.; Kaisner, J. Robot Inclusive Space challenge: A design initiative. In Proceedings of the 6th IEEE Conference on Robotics, Automation and Mechatronics (RAM), Manila, Philippines, 12-15 November 2013; pp. 73-78.

24. Mohan, R.E.; Tan, N.; Tjoelsen, K.; Sosa, R. Designing the robot inclusive space challenge. Dig. Commun. Netw. 2015, 1, 267-274.

25. IDC Robocon Official Website. Available online: http://www.idc-robocon.org/e/index (accessed on 1 June 2016).

26. Yamakita, M. Engineering Design Education via IDC Robot Contest. In Proceedings of the IFAC Symposium on Advances in Control Education, Kumamoto City, Japan, 21-23 October 2009; Volume 8, pp. 227-232.

27. Toh, C.A.; Miller, S.R.; Kremer, G.E.O. The Impact of Team-Based Product Dissection on Design Novelty. J. Mech. Des. 2014, 136, doi:10.1115/1.4026151.

28. Austin-Breneman, J.; Honda, T.; Yang, M.C. A Study of Student Design Team Behaviors in Complex System Design. J. Mech. Des. 2012, 134, doi:10.1115/1.4007840.

29. Grover, R.; Krishnan, S.; Shoup, T.; Khanbaghi, M. A competition-based approach for undergraduate mechatronics education using the arduino platform. In Proceedings of the Interdisciplinary Engineering Design Education Conference (IEDEC), Santa Clara, CA, USA, 3 March 2014; pp. 78-83.

(C) 2016 by the authors; licensee MDPI, Basel, Switzerland. This article is an open access article distributed under the terms and conditions of the Creative Commons Attribution (CC-BY) license (http://creativecommons.org/licenses/by/4.0/). 